\title{
Consideration of a comprehensive animal model of addiction: the limitations of modeling a counterfeit condition
}

\author{
Stephen T. Tiffany
}

Received: 31 December 2013 / Accepted: 29 January 2014 /Published online: 29 May 2014

(C) Springer-Verlag Berlin Heidelberg 2014

The literature on addiction is so massive that it can crush even the most seasoned scientist. Thus, any attempt to fully consolidate and integrate the knowledge generated by the drug addiction field over 40 years of research is unquestionably ambitious but almost certainly unworkable. Unsurprisingly, though the paper of Piazza and Deroche-Gamonet (2013) is an expansive and elaborate treatise on addictive behavior, it is not nearly as comprehensive as promised. What seems to be notably lacking is a full consideration of the human condition of addiction.

At its heart, this paper describes a straightforward theory. According to the authors, addiction proceeds in three phases with increasingly fewer people progressing to each successive phase. Transitions from one phase to the next come about from interactions between individual vulnerabilities and patterns of drug exposure. Phase 1 is characterized by experimentation with drugs, phase 2 by more sustained and prolonged drug use, and phase 3 by loss of control over drug use. The vulnerabilities for movement from phase 1 to 2 are hypothesized to be different from the vulnerabilities assumed to be necessary for movement from phase 2 into phase 3 . The authors also theorize that the psychobiological mechanisms responsible for drug use at each phase are distinct, with phase 1 controlled by "normal learning processes," phase 2 dominated by incentive sensitization and a shift in hedonic set point, and phase 3 characterized by a loss of synaptic plasticity. The idea that addiction is categorically distinct from nonaddictive drug use stands in contrast to the common assumption that addiction is a dimensional construct with those engaged in very heavy levels of use only quantitatively, but

S. T. Tiffany $(\bowtie)$

Department of Psychology, University at Buffalo, The State University of New York, 206 Park Hall, Buffalo, NY 14260, USA

e-mail: stiffany@buffalo.edu not qualitatively, different from less experienced users (Goedeker and Tiffany 2008; Tiffany et al. 2004).

The authors also write extensively about issues that are not core to their theory. Some of their assertions seem almost selfevidently true. For example, the authors note that many animal studies are of such short duration and provide so limited exposure to drugs that they are unlikely to shed much light on the processes at play in chronic drug users with a history of multiple years of use. That is, despite de rigueur sections of many papers that proclaim the clinical significance of the research, a great deal of animal research may be irrelevant to understanding end-stage addiction.

Other assertions are more discursive. Critically, the authors declare that their theory demonstrates that drug addiction is a "true psychiatric disease." This is a questionable claim on many levels. First, how can an unvalidated theory demonstrate that a condition is a disease-that would push the limits of what any theory might accomplish. Further, I am unaware that any standards have yet been established for identifying a behavioral disorder as a disease as opposed to non-disease condition, and the paper provides no useful elucidation of this ontological issue. Finally, the argument that addiction is a disease because it may share some fundamental features with other established psychiatric diseases (e.g., anxiety disorders and depression) is not convincing, as these disorders are no more established as diseases (other than by medical fiat) than is addiction. As unsatisfying as it may be, Jellinek's (1960) assertion that "A disease is what the medical profession recognizes as such," may be the best that we can do if we want to describe addiction as a disease.

The authors also claim that a shift from conventional drugcentered theories to their individual-centered approach along with the adoption of their idea that addiction is a "true psychiatric disorder" would dramatically humanize our treatment of addiction and profoundly alter our societal approach to addiction. There are many arguments for humanizing our 
approach to addiction, but widespread adoption of the idea that addiction is a psychiatric disease may not have the salutary impact the authors envisage. Psychiatric disorders are stigmatizing; there is little reason to believe that the "disease" of addiction would be viewed by the public with less disapproval than most other psychiatric conditions.

What about the theory itself? Is there anything here that seems implausible or inconsistent with extant evidence? The theory repurposes, both in its particulars as well as in its broad sweep, many concepts, theories, and hypotheses that have been forwarded previously by others. These include stages of addiction, stage-specific vulnerabilities, incentive learning, habit learning, allostasis, dopaminergic involvement in reward systems, and neuroanatomical substrates of reward and inhibitory control. The fresh contribution of this theory is the hypothesized distinct break, both behavioral and mechanistic, between the proposed second and third stages of addiction. The authors make a great deal of the third stage, the so-called full addiction phase, as characterized uniquely by loss of control over drug use. Loss of control is a highly controversial concept in the addictions literature, as it suggests an absolute surrender to drug use with the implication that users are completely insensitive to rewards for not using drugs. We know that is not true-even people with extraordinarily entrenched patterns of drug use will decrease their substance use if given reinforcement for abstinence (Prendergast et al. 2006)-in fact, we have known this for a long time (Mendelson and Mello 1966; Nathan et al. 1970). Perhaps, a better descriptor is that severe forms of addiction are characterized by diminished control over drug use, that is, control may be best thought of as a dimensional construct with end-stage addiction on the far end of that continuum. The possibility that control is dimensional would severely undermine the authors' proposition that there is a sharp demarcation between the second and third phases of addiction.
Nearly all of the evidences for a categorically distinct third phase of addiction as envisioned by the authors come from rat studies with cocaine administration. That may be a very thin reed on which to base a "general theory of addiction." Of course, time and targeted research will tell us whether this is a viable model of rodent behavior and, if it is, whether the findings in rats will teach us much about end-stage addiction in humans. If the ultimate goal is to model loss of control, researchers may want to more clearly define that construct in human addiction. They may find that, in humans, the concept of control over drug use is much more complicated, behaviorally and biologically, than the caricature modeled by this theory.

Conflict of interest The author declares no conflict of interest in the preparation of this commentary.

\section{References}

Goedeker KC, Tiffany ST (2008) On the nature of nicotine addiction: a taxometric analysis. J Abnorm Psychol 117:896-909

Jellinek EM (1960) The disease concept of alcoholism. Hillhouse, New Haven

Mendelson JH, Mello NK (1966) Experimental analysis of drinking behavior of chronic alcoholics. Ann N Y Acad Sci 133:828-845

Nathan PE, Titler NA, Lowenstein LM, Solomon P, Rossi AM (1970) Behavioral analysis of chronic alcoholism. Arch Gen Psychiatry 22: 419-430

Piazza PV, Deroche-Gamonet V (2013) A multistep general theory of transition to addiction. Psychopharmacology 229:387-413

Prendergast M, Podus D, Finney J, Greenwell L, Roll J (2006) Contingency management for treatment of substance use disorders: a meta-analysis. Addiction 101:1546-1560

Tiffany ST, Conklin CA, Shiffman S, Clayton RR (2004) What can dependence theories tell us about assessing the emergence of tobacco dependence? Addiction 99(S1):78-86 